\title{
How wettability controls nanoprinting
}

\author{
Juan Carlos Fernández-Toledano, ${ }^{1}$ Bertrand Braeckeveldt, ${ }^{1}$ Marco Marengo, ${ }^{2}$ and Joël De Coninck ${ }^{1}$ \\ ${ }^{1}$ Laboratory of Surface and Interfacial Physics (LPSI), \\ Department of Physics, University of Mons, Mons, Belgium \\ ${ }^{2}$ Advanced Engineering Centre, University of Brighton, Brighton BN2 4GJ, U.K.
}

\begin{abstract}
Using large scale molecular dynamics (MD) simulations we study in detail the impact of nanometer droplets of low viscosity on flat substrates versus the wettability of the solid plate. The comparison between the MD simulations and different macroscopic models reveals that most of these models do not correspond to the simulation results at the nanoscale in particular for the maximal contact diameter during the nanodroplet impact $\left(D_{\max }\right)$. We have developed a new model for $D_{\max }$ which is in agreement with the simulation data and also takes into account the effects of the liquid-solid wettability. We also propose a new scaling for the time required to reach the maximal contact diameter, $t_{\max }$ with respect to the impact velocity which is also in agreement with the observations. With the new model for $D_{\max }$ plus the scaling found for $t_{\max }$, we present a master curve collapsing the evolution of the nanometer drop contact diameter during impact for different wettabilities and different impact velocities. We believe our results may help to design better nanoprinters since they provide an estimation of the maximum impact velocities required to obtain a smooth and homogenous coverage of the surfaces without dry spots.
\end{abstract}

PACS numbers:

More and more applications demand a detailed understanding of nanodroplets such as for instance 3D nanoprinting, nanomedicine, phase-change cooling using nanosprays and microdeposition processes [1-3]. Remarkable achievements have been obtained rather recently by printing organic molecules at the nanoscale [4]. The interest of such technology is clear, it is flexible, inexpensive, fast, addressable and scalable. Most of the practical applications are based on the idea that a nanodrop is used as a carrier of some biological elements or nanoparticles of one sort or another. It then remains to evaporate the carrier, the solvent of the nanodrop, to have access to a patterned surface with interesting functionalities. The idea is simple and the technology is already mature enough to make it work [4]. Now, depending on the type of solvent, the shape of the nanodrop will vary not only with time but also with the impact speed of the nanodrop. This is where wetting and impact dynamics become critical. It is rather obvious that for small volumes the evaporation time for the solvent will be fast. If it is faster than the time required for the liquid to spread, we will end up with different structures leading to different properties. It is therefore mandatory to understand how wettability affect impact at such a small scale since wettability and evaporation are also intimately related [5].

Due to the huge number of applications involved, drop impact has been intensively studied in the past years as, for example, the modeling of the influence of the drop size on the time evolution of the dynamic contact angle and contact radii [6], the study of the transition between bounce/stick/splash of nanodroplets $[7,8]$, the impact of nanodroplets on textured surfaces [9], droplet impact over inclined substrates studied experimentally [10] or by using molecular dynamics techniques [11], experimen- tal studies of drop impact over a moving substrate [12], etc. Given the strong deformation of the droplet during impact, especially for cases with relatively high impact velocities, the evaluation of the dynamic contact angle is not very accurate in comparison with the measurement of the contact diameter of the drop. Furthermore, different models have been proposed to characterize the maximum spreading diameter of a drop at macroscale [13-18]. Most of these models are either empirical or based on energy balance plus viscous dissipation but not all take into account the wetting properties between the liquid and the solid phases [14, 16, 18]. Moreover, these models have been compared with experiments at a macroscopic scale but their validity at the nanometric scale, which is essential for many of the applications described above, is still unknown.

In this study we use large scale molecular dynamics (MD) simulations using LAMMPS software [19] to analyze the impact of nanodroplets with a typical diameter of $D_{0}=20.3 \mathrm{~nm}$ onto flat substrates. MD allows us to tune fundamental parameters like strength of interactions between atoms in order to explore the details of nanoprinting. By studying how the drops behave versus their equilibrium contact angles and the impact speeds, we will get a better understanding of impact for nanoprinting leading potentially to interesting optimization.

All the atoms interact between each other via a pairwise Lennard-Jones (LJ) potential $V_{i j}=$ $-4 C_{A B} \epsilon\left[(\sigma / r)^{6}-(\sigma / r)^{12}\right]$ where $r$ is the distance between atoms, $\epsilon=k_{B} T$ is the depth of the potential, $\sigma=0.35 \mathrm{~nm}$ is the effective atom diameter and $T=$ $33 \mathrm{~K}$ is the temperature and atom mass is $m_{0}=12$ $\mathrm{g} / \mathrm{mol}$. The selected values of $\sigma$ and $m_{0}$ are selected to model carbon-like atoms. By tunning the value of $C_{A B}$ (coupling between type "A" and type "B" atoms) 
we are able to change the strength of the interaction between the two types of atoms. We keep constant the value of the solid-solid and liquid-liquid coupling to $1.0\left(C_{S S}=C_{L L}=1\right)$ and we only tune the liquidsolid coupling $\left(C_{L S} \in[0.4,0.8]\right)$ which enables to explore different wettabilities with equilibrium contact angles $\theta^{0} \in\left[132^{\circ}, 78^{\circ}\right]$ and thus study in details the importance of this parameter at the nanoscale. The liquid is made of 10648 linear molecules of 8-atoms each. Atoms inside a molecule are linked through a FENE potential $V_{F}=-0.5 \kappa R_{0}^{2} \ln \left[1-\left(r / R_{0}\right)^{2}\right]$ where $r$ is the distance between atoms, $\kappa=12.25 \epsilon / \sigma^{2}$ and $R_{0}=1.4 \sigma$ is the maximum extension length. The solid substrate is modeled as 299040 atoms distributed in a cubic lattice over a disk with a radius of $69.8 \mathrm{~nm}$ and three atoms layers where the lattice parameter is equal to the location of the minima of the Lennard-Jones potential, i.e. $2^{1 / 6} \sigma \approx 0.39$ $\mathrm{nm}$. The corresponding atoms are allowed to vibrate around an equilibrium position by introducing an harmonic potential which bonds the solid atoms to their initial positions. The corresponding harmonic potential is $V_{h}=200 \epsilon\left(r-r_{0}\right)^{2}$ where $r_{0}$ represents the equilibrium position of the solid atoms in the lattice. The cutoff of all the interactions is set to $2.5 \sigma$ and the timestep between each interaction evaluation was fixed to 5 fs. For simplicity, we have not consider the presence of a surrounding fluid which seems not to have a strong influence during the spreading process $[7,20]$ although it can be critical for splashing [20].

Each simulation consists of two steps. In the first one, we equilibrate the system with the liquid atoms initially distributed in a square box far away from the influence of the substrate. During the equilibration the temperature is kept constant by rescaling all the velocities. The equilibrium stage is achieved when the energy is constant versus time and the droplet shows a spherical cap shape of constant diameter $D_{0}=(20.28 \pm 0.24)$ $\mathrm{nm}$. After the equilibration step, the thermostat of the liquid is removed in order to enable thermal exchanges between the two phases allowing thus the liquid to dissipate energy within the solid. The drop is also translated just above the substrate to reduce the distance to cross before impact. Finally, an initial impact velocity $V_{i m p} \in[10,200] \mathrm{m} / \mathrm{s}$ in the direction perpendicular to the solid is set to the drop in order to initiate the impact. By using standard methods [21] we compute the liquid surface tension $\gamma_{L}=(2.85 \pm 0.56) \mathrm{mN} / \mathrm{m}$, the viscosity $\eta_{L}=(0.284 \pm 0.004) \mathrm{mPa} \cdot \mathrm{s}$ and the liquid density $\rho_{L}=(386 \pm 5) \mathrm{kg} / \mathrm{m}^{3}$. The model is thus rather simple but it has been used previously to study drop spreading [22] and liquid bridges [23] where this technique has been shown to be able to reproduce adequately macroscopic properties such as Laplace pressure and Young's law. We compute the instantaneous contact diameter as $D(t)=2 r_{c}(t)$ where $r_{c}(t)$ is the radial distance with respect the mass center of the drop where the density of the liquid in contact with the plate drops at $50 \%$ of the value corresponding to the central part. Then, $D_{\max }$ corresponds just to the maximum value of $D(t)$ measured during the impact.

As a preliminary test, we study the increase of the liquid temperature $\Delta T$ with the impact velocity $V_{i m p}$ which will introduce an increment of the kinetic energy on the drop that is converted to heat at impact, i.e. $\Delta T=V_{i m p}^{2} / 2 c_{v}$ where $c_{v}$ is the specific heat of the liquid. The specific heat can be measured from the energy fluctuations [24] in an independent simulation leading to the value of $c_{v}=(2533 \pm 380) \mathrm{J} / \mathrm{kg} \cdot \mathrm{K}$. The increment of the temperature of the liquid can be measured in the simulations versus the impact velocities and it has been observed that these values are independent on the considered wettability and are compatible with the expected theoretical value which corroborates the validity of the simulation methods.

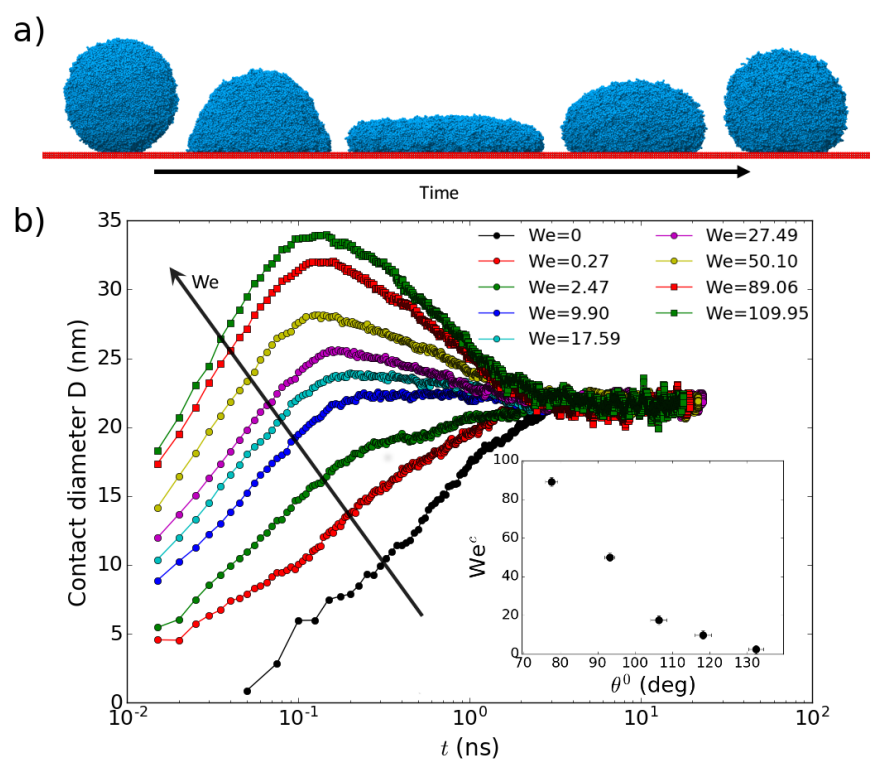

FIG. 1: a) Snapshot of the simulations for $C_{L S}=0.4\left(\theta^{0}=\right.$ $\left.132^{\circ}\right)$ and $\mathrm{We}=109.9$ at different times. b) Droplet contact diameter versus time for various impact velocities for $C_{L S}=$ 0.6 corresponding to the equilibrium contact angle $\theta^{0}=106^{\circ}$.

First, we study the effect of the variation of the impact velocity $V_{i m p}$ on the dynamics of the droplet contact diameter. In order to compare our results with the different models presented in the literature it is convenient to express $V_{i m p}$ in dimensionless Weber number defined as We $=\rho_{L} D_{0} V_{i m p}^{2} / \gamma_{L}$ (the ratio of the liquid inertia and the surface tension) which it is tuned between $\mathrm{We}=0.27$ $\left(V_{i m p}=10 \mathrm{~m} / \mathrm{s}\right)$ to $\mathrm{We}=109.9\left(V_{i m p}=200 \mathrm{~m} / \mathrm{s}\right)$. Figure 1a shows different snapshots during the impact for $C_{L S}=0.4\left(\theta^{0}=132^{\circ}\right)$ and $W e=109.9$. In Fig. $1 \mathrm{~b}$ we show the effect of the Weber number on the spreading dynamics for $C_{L S}=0.6\left(\theta^{0}=106^{\circ}\right)$. By increasing the Weber number, the maximum contact diameter $D_{\max }$ 
rises within a considerable range $(\sim 34 \mathrm{~nm})$ for a droplet of initial diameter of $20.3 \mathrm{~nm}$ which is an expected result observed experimentally [14-16, 25, 26]. However, there are impact velocities for which the droplet never overcomes the equilibrium contact diameter $D_{e q}$. This can be seen in Fig. 1b for $\mathrm{We}<30$. Let us here stress that this limit depends on the wettability of the plate. Therefore, there is a threshold in the Weber number $\mathrm{We}^{c}$ to reach a maximum spreading diameter larger than the equilibrium one which is shown in the inset of Fig. 1b for the different equilibrium contact angles studied. It is clear that this critical impact velocity $\mathrm{We}^{c}$ strongly depends on the wettability. However, as it has been reported by other authors $[15,25]$, when $\mathrm{We}>\mathrm{We}^{c}$ the time evolution of $D(t)$ until $D_{\max }$ is basically driven by inertia and the impact process is relatively insensitive to the surface wetting properties and we get an increment of $D_{\max }$ when the solid-liquid affinity is decreased as has been observed at low impact velocities (We $<30$ ). Let us here point out that our results cannot be directly extrapolated to predict the properties of real superhydrophobic surfaces. Indeed, for such surfaces, superhydrophobicity is due to roughness which has not been introduced here. Therefore, the very low values of $\mathrm{We}^{c}$ for $\theta^{0}>120^{\circ}$ does not necessarily imply that the impacts will be inertia driven in these substrates.

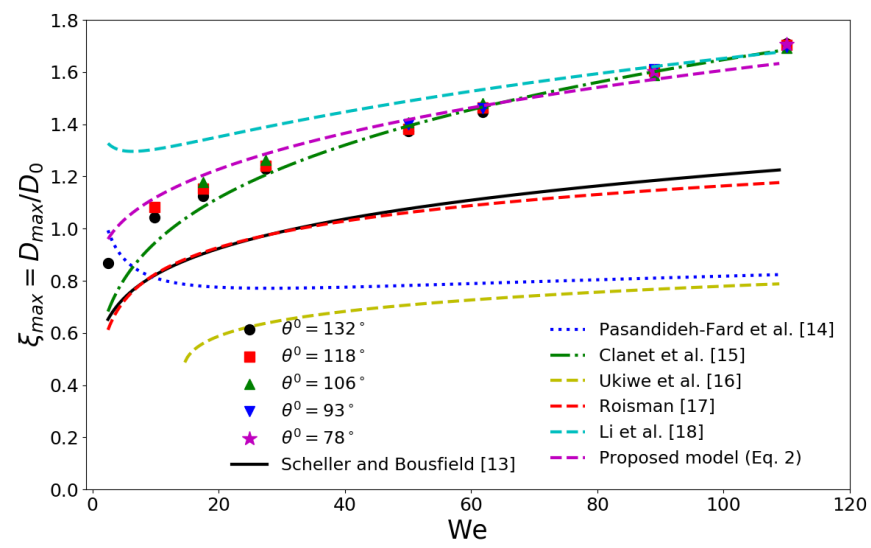

FIG. 2: $\xi_{\max }=D_{\max } / D_{0}$ versus the Weber number for all liquid-solid affinities studied and for $\mathrm{We}>\mathrm{We}^{c}$, i.e. when $D_{\max }>D_{\text {eq }}$. The lines represent the different models proposed in the literature.

Figure 2 shows the values of $\xi_{\max }=D_{\max } / D_{0}$ for the different impact velocities (different We) and various liquid-solid equilibrium contact angles. $D_{\max }$ is here computed by a gaussian fitting around the peak location on $D(t)$. For the lower impact velocity, there is an increment of $\xi_{\max }$ as the solid-liquid affinity is increased but this difference dissapears at higher impact velocities. However, once $D_{\max }$ is reached we observe in the simulations that the wettability modify completely the dewetting process until equilibrium which can be very impor- tant to evaporate the solvant used to carry nanoparticles for instance.

Many correlations and models have been proposed in literature $[13-15,17,28-31]$ to predict time evolution of the maximum spreading factor $\xi_{\max }$. These correlations very rarely take into account the wettability effect and they have been compared so far with experiments at the macroscale. In Fig. 2 we observe that the simulated $D_{\max } / D_{0}$ can be fitted with the power law $\xi_{\max } \propto \mathrm{We}^{1 / 4}$ introduced by Clanet et al. [15] for low viscous liquids based on the effective acceleration experienced by the drop during its impact. Clanet considers the intial kinetic energy due to the drop velocity is not only transformed to surface energy, but also to internal kinetic energy. However, the value of this internal energy is not clear and therefore, the complete description of $\xi_{\max }$ versus We without fitting parametes remains missing. As it is shown in Fig. 2, the semi-empirical models derived from the energy balance $[14,16,32]$ cannot described properly the results for our low viscous liquid at the nanoscale. The origin of this discrepancy has certainly be located in the approximation used for the dissipation contribution which considers that the velocity gradient only exist in the boundary layer adjacent to the liquid-solid interface [14]. Different approximations have been proposed $[14,16,32]$ to describe the viscous dissipation in the impact of droplets at the macroscale. The validity of these approaches is however uncertain at the nanoscale. Based on the analysis of the velocity distribution in MD simulations of water nanodroplets impact, Li et al. [18] found that the velocity gradient exist not only in the boundary layer but that it is quite uniform in the droplet. With these considerations, they propose that the viscous dissipation for impact of nanodroplets can be written as $W=\pi \eta_{L} D_{0}^{2} V_{i m p}\left(\xi_{\max }^{2}-2 / 3\right) / 4$. However, as in the case of Pasandideh-Fard et al. [14], they underestimate the liquid-vapor interface at the maximum spreading state in the energy balance equation and the resulting prediction of $\xi_{\max }$ does not describe properly our simulation results as it is shown in Fig. 2.

Injecting the more precise approximation for the interfacial areas from Ref. [16] in the calculations, we obtain a new equation from the energy balance (see Appendix I for details):

$$
3\left(\mathrm{Ca}+1-\cos \theta^{0}\right) \xi_{\max }^{3}-(\mathrm{We}+12+2 \mathrm{Ca}) \xi_{\max }+8=0
$$

Where $\mathrm{Ca}=\eta_{L} V_{i m p} / \gamma_{L}$ is the capillary number associated to the impact velocity. Equation 1 has an analytical solution

$$
\xi_{\max }=2 \sqrt{-\frac{p}{3}} \cos \left(\frac{1}{3} \arccos \left(-\frac{q}{2} \sqrt{-\frac{27}{p^{3}}}\right)\right)
$$

where $p=-(\mathrm{We}+12+2 \mathrm{Ca}) /\left[3\left(\mathrm{Ca}+1-\cos \theta^{0}\right)\right]$ and $q=8 /\left[3\left(\mathrm{Ca}+1-\cos \theta^{0}\right)\right]$.

Figure 3 a shows $D_{\max }$ measured from the simulation versus the prediction obtained from Eq. 2 where symbols 

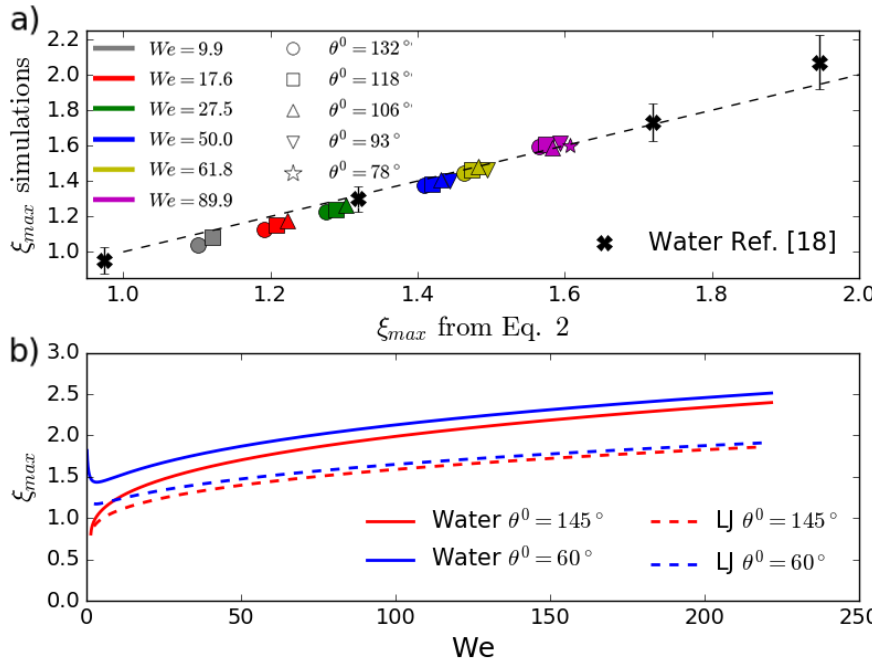

FIG. 3: a) $\xi_{\max }=D_{\max } / D_{0}$ versus the Weber number for all liquid-solid affinities studied and the prediction from Eq. 2 for $\theta^{0}=\left[132^{\circ}, 78^{\circ}\right]$. b) Simulation results for impact of water nanodroplets from Ref. [18] and the model from Eq. 2 for water (continuous lines) and LJ (dashed lines) liquid with $\theta^{0}=\left[145^{\circ}, 60^{\circ}\right]$

and colors represent equilibrium contact angles and impact velocities, respectively. A good agreement between theory and simulation is observed. Also, we model predicts an increment of $D_{\max }$ when we increase the affinity between the liquid and the solid for low impact velocities but the wettability effect becomes negligible for larger We. The proposed model is thus able to predict quite well not only the effect of the impact velocity but also the role of the wettability on $D_{\max }$.

The lack of experimental results of nanodroplets impact impedes the comparison of Eq. 2 with real data. Nevertheless, we can validate the proposed model with the simulated results of water nanodroplets in platinum performed in Ref. [18] which can be also reproduced with the proposed model as it is shown in Fig. 3a. Equation 2 also reveals that the role of the wettability depends on liquid properties. As an example. in Fig. 3b we show the $\xi_{\max }$ predicted by Eq. 2 for two liquids, our LennardJones liquid and water, and two different substrate wettabilities. Interestingly, the effect of the wettability becomes more important for water due to its larger value of surface tension. Then, Eq. 2 can be used in nanoprinting to predict the corresponding extension $D_{\max }$ from an initial droplet of diameter $D_{0}$ for any wettability $\theta_{0}$ at any impact speed $V_{i m p}$.

Let us now consider the time $t_{\max }$ at which the drop impacting the substrate reaches $D_{\max }$ whenever $D_{\max }>$ $D_{e q}$ corresponding thus to the case where $\mathrm{We}>\mathrm{We}^{c}$. For a precise detection of $t_{\max }$ we select a region around $D_{\max }$ and we fit a gaussian distribution. We identify the central value of this Gaussian with $t_{\max }$. The representation of $t_{\max }$ can be modeled as a power law: $t_{\max }(\mathrm{ns})=(0.319 \pm 0.016) \mathrm{We}^{-(0.196 \pm 0.014)}$ which basically corresponds to the scaling $t_{\max } \propto \mathrm{We}^{-1 / 5}$. This power-law dependence of $t_{\max }$ has been observed experimentally at the macroscale with very similar exponents: Antonini et at. [27] obtain $t_{\max } \propto \mathrm{We}^{-0.25}$ and Roux et al. $[26] t_{\max } \propto \mathrm{We}^{-0.32}$. With the resolution we have in the determination of $t_{\max }$ it is not possible to establish any influence of the wettability neither of the macro/nanoscale in the time required to achieve $D_{\max }$ that appears for $\mathrm{We}>\mathrm{We}^{c}$.

Once we have obtained $D_{\max }$ from Eq. 2 and with the power law $t_{\max } \propto \mathrm{We}^{-1 / 5}$ it is possible to transform the temporal evolution of the base diameter $D(t)$ to have dimensionless units by dividing $D(t)$ and $t$ by $D_{\max }$ and $\mathrm{We}^{-1 / 5}$, respectively. With this transformation, in Fig. 4 it can be seen that all the data collapse into one universal curve before reaching the maximum spreading. Furthermore, it works not only for different impact velocities but also for different substrate wettabilities (different couplings $C_{L S}$ ) providing a good tool for nanoprinting. We do not reproduce all these data here for clarity. Therefore, it is possible to anticipate the temporal evolution of $D(t)$ at all impact velocities from a measurement of $D(t)$ at a single $V_{i m p}$.

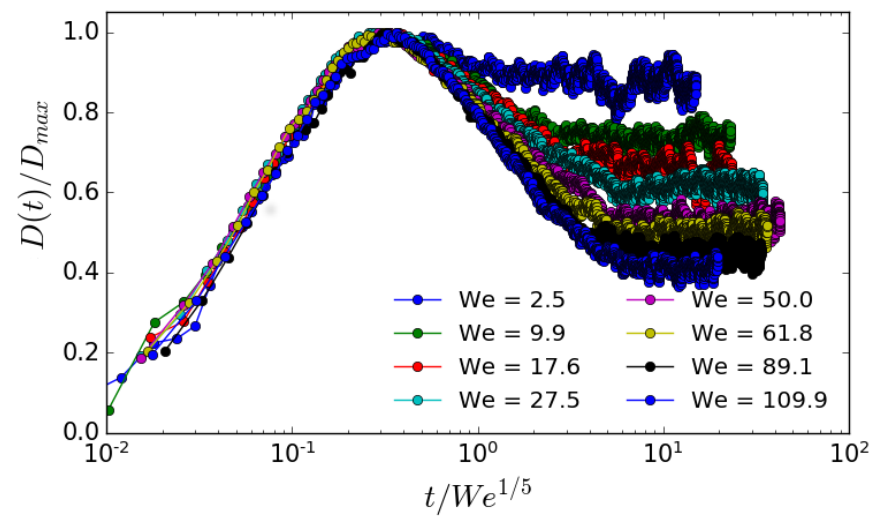

FIG. 4: Collapsing of $D / D_{\max }$ vs. $t / \mathrm{We}^{-1 / 5}$ in a single curve for $\theta^{0}=132^{\circ}$.

To conclude, in this study we have shown that molecular dynamics is an interesting tool to analyse underlying mechanisms of drop impact for nanoprinting. It allows us to probe molecular displacements and interactions during the whole process. We have shown that by increasing the impact velocity over a threshold value $W e^{c}$, we increase the maximum spreading diameter of the droplet $D_{\max }$. We have also shown that $t_{\max }$, i.e. the time required to reach this maximum spreading, decreases as we increase the impact velocity. Moreover, we have studied the influence of the wettability over $D_{\max }, t_{\max }$ and $\mathrm{We}^{c}$. We have shown that the threshold velocity $\mathrm{We}^{c}$ decreases as the contact angle increases. Also, we have found that $D_{\max }$ increases as the equilibrium contact angle between 
the liquid and the solid $\theta^{0}$ is decreased for low impact velocities $\left(\mathrm{We}^{c}<\mathrm{We}<30\right)$ but it seems to be independent on $\theta^{0}$ when $\mathrm{We}>\mathrm{We}^{c}>30$. However, $t_{\max }$ seems to be independent on the wettability for all equilibrium contact angles studied. We have proposed a new model to predict $\xi_{\max }=D_{\max } / D_{0}$ for nanodroplets impact based on energy balance that successfully reproduce the simulation results and even the effect of changes in the wettability on $D_{\max }$ for low impact velocities. This model reveals the role of the substrate wettability which could become important depending on the liquid properties like surface tension and viscosity. Also, we have shown that $t_{\max } \propto \mathrm{We}^{-1 / 5}$, a scalling similar to the experimental observations. With the proposed models for $D_{\max }$ and $t_{\max }$ we have seen that we can overlap in a master curve the evolution of the contact diameter $D(t)$ till $D_{\max }$ for all the impact velocities and all wettabilies used in this study. An interesting consequence of this work is that nanoprinting becomes predictable since we can estimate $t_{\max }$ as a function of the impact velocity and from Eq. 2 , we can predict $D_{\max }$. However, experimental work in nanodroplets impact is required to validate the approach proposed.

The authors thank the European Space Agency (ESA) and the Belgian Federal Science Policy Office (BELSPO) for their support in the framework of the PRODEX Programme. This research has also been partially funded by UMONS. Computational resources have been provided by the Consortium des Equipements de Calcul Intensif (CECI), funded by the Fonds de la Recherche Scientifique de Belgique (F.R.S.-FNRS) under Grant No. 2.5020.11

[1] A. Ishijima, K. Minamihata, S. Yamaguchi, S. Yamahira, R. Ichikawa, E. Kobayashi, M. Iijima, Y. Shibasaki, T. Azuma, N. T., et al., Sci. Rep 7, 44077 (2017).

[2] J. Ventrici de Souza, Y. Liu, S. Wang, P. Dörig, T. L. Kuhl, J. Frommer, and G.-Y. Liu, J. Phys. Chem.: B 122, 956 (2018).

[3] W. Wu, H. Bostanci, L. Chow, S. Ding, Y. Hong, M. Su, J. Kizito, L. Gschwender, and C. Snyder, Int. J. Heat Mass Transf. 54, 2715 (2011).

[4] C. U. Hail, C. Höller, K. Matsuzaki, P. Rohner, J. Renger, V. Sandoghdar, D. Poulikakos, and H. Eghlidi, Nat. Commun 10, 1880 (2019).
[5] D. Brutin and V. Starovc, Chem. Soc. Rev. 47, 558 (2018).

[6] N. Sedighi, S. Murad, and S. K. Aggarwal, Fluid Dyn. Res. 42, 035501 (2010).

[7] J. Koplik and R. Zhang, Phys. Fluids 25, 022003 (2013).

[8] A. Yarin, Annu. Rev. Fluid Mech. 38, 159 (2006).

[9] R. Zhang, S. Farokhirad, T. Lee, and J. Koplik, Phys. Fluids 26, 082003 (2014).

[10] J. Hao, J. Lu, L. Lee, Z. Wu, G. Hu, , and J. M. Floryan, Phys. Rev. Lett. 122, 054501 (2019).

[11] A. Delcorte and B. J. Garrison, Physics Research B. 303, 179 (2013).

[12] H. Almohammadi and A. Amirfazli, Langmuir 33, 59575964 (2017).

[13] B. L. Scheller and D. W. Bousfield, AIChE Journal 41, 1357 (1995).

[14] M. Pasandideh-Fard, Y. M. Qiao, S. Chandra, and J. Mostaghimi, Phys. Fluids 8, 650 (1996).

[15] C. Clanet, C. Béguin, D. Richard, and D. Quéré, J. Fluid Mech 517, 199 (2004).

[16] C. Ukiwe and D. Y. Kwok, Langmuir 21, 666 (2005).

[17] I. V. Roisman, Phys. Fluids 21, 052104 (2009).

[18] x.-H. Li, X.-X. Zhang, and M. Chen, Phys. Fluids 27, 052007 (2015).

[19] S. Plimpton, J. Comput. Phys. 117, 1 (1995).

[20] L. Xu, W. W. Zhang, and S. R. Nagel, Phys. Rev. Lett. 94, $184505(2005)$

[21] M. P. Allen and D. J. Tildesley, Computer Simulation of Liquids (Clarendon Press, New York, NY, USA, 1989), ISBN 0-19-855645-4.

[22] E. Bertrand, T. D. Blake, and J. De Coninck, J. Phys.: Condens. Matter 21, 464124 (2009)

[23] J.-C. Fernandez-Toledano, T. D. Blake, P. Lambert, and J. De Coninck, Adv Colloid Interface Sci 245, 102 (2017).

[24] H. B. Callen, Thermodynamics and an introduciton to thermostatistics (Wiley, New York, 1985).

[25] R. Rioboo, M. Marengo, and C. Tropea, Exp Fluids 33, $112(2002)$.

[26] D. C. D. Roux and J. J. Cooper-White, J. Colloid Interface Sci. 277, 424 (2004).

[27] C. Antonini, A. Amirfazli, and M. Marengo, Phys. Fluids 24, 102104 (2012).

[28] T. Mao, D. C. S. Kuhn, and H. Tran, AIChE Journal 43, 2169 (1997).

[29] H.-Y. Kim and J.-H. Chun, Phys. Fluids 13, 643 (2001).

[30] J.-P. Delplanque and R. H. Rangel, J. Mater. Sci. 32, 1519 (1997).

[31] P. Attané, F. Girard, and V. Morin, Phys. Fluids 19, 012101 (2007)

[32] S. Chandra and C. T. Avedisian, Proc. Roy. Soc. London, Ser. A 432, 13 (1991). 